\title{
Thiamine Therapy and Refeeding Syndrome in Extremely Low Birth Weight Infants
}

JCR Shaker Rouba, Charafeddine Fatme, Charafeddine Lama

Department of Pediatrics and Adolescent Medicine, American University of Beirut Medical

Center, Beirut, Lebanon.

Abstract:

An extremely low birth weight (ELBW) infant with severe intrauterine growth restriction (IUGR), born at $29^{+1}$ weeks of gestation, developed severe electrolyte disturbances with metabolic acidosis three days after initiation of total parenteral nutrition (TPN). Correction of acidosis and electrolyte imbalance was only achieved after addition of thiamine infusion. This case-report highlights the importance of thiamine therapy in correcting the metabolic acidosis and electrolyte imbalance associated with refeeding syndrome in infants with severe IUGR.

Key words: Acidosis, Fetal Growth Retardation, Infant, Refeeding Syndrome, Thiamine.

\section{Introduction}

Refeeding syndrome is a constellation of fluids and electrolytes dysregulation that occurs upon initiation of enteral or parenteral nutrition following a prolonged period of malnutrition or starvation. This syndrome has been extensively described in patients with anorexia, post-surgical adult and pediatric population and in the intensive care setting [1-4]. It has been recently reported in the neonatal population occurring in very low birth (VLBW) infants with intrauterine growth restriction (IUGR) [5-6], typically born to mothers with preeclampsia [7]. The electrolytes disturbances characteristics of the refeeding syndrome are hypophosphatemia, hypokalemia, hyperglycemia and hypomagnesemia. The underlying mechanism is attributed to increased glucose metabolism after initiation of feeding, which leads to increased phosphate usage for ATP production. This increased demand for phosphorus depletes serum stores of phosphorus as well as magnesium $[1,3,7,8]$. During chronic malnutrition, in addition to glucose and glycogen stores depletion causing a metabolic adaptation where energy is derived from lipolysis and ketone production, there are significant vitamin deficiencies, in particular thiamine [Fig.1] [7]. Upon refeeding, the relative excess of glucose delivery in a system now adapted to fat metabolism, results in hyperglycemia and secondary hyperinsulinemia [7]. Increased insulin leads to intracellular shift of phosphorus and potassium [9].

For this reason, in severely malnourished children $[10,11]$ and in adults receiving exclusively TPN as nutrition, it is recommended to start feeding

\section{Corresponding Author: Dr. Lama Charafeddine}

Email: Ic12@aub.edu.lb

Received: February 19, 2016 | Accepted: May 10, 2016 | Published Online: July 5, 2016

This is an Open Access article distributed under the terms of the Creative Commons Attribution License (creativecommons.org/licenses/by/3.0)

Conflict of interest: None declared | Source of funding: Nil | DOl: http://dx.doi.org/10.17659/01.2016.0074 
with slow and gradual caloric intake and to supplement with thiamine which is essential for rapid resolution of acidosis occurring with the refeeding syndrome $[8,12,13]$. In neonates, thiamine deficiency has been reported to cause severe fatal metabolic acidosis during the inadvertent administration of TPN lacking thiamine supplementation. In those cases the addition of thiamine resulted in rapid resolution of metabolic acidosis [12]. We are reporting this case to illustrate the importance of thiamine administration in correcting not only metabolic acidosis, but also the electrolyte imbalance seen in refeeding syndrome in neonates with IUGR and small for gestational age (SGA).

\section{Case Report}

This is a 487 gram preterm female infant product of $29^{+1}$ weeks of gestation, born to a 33 year-old mother G2P1L1A0, who is toxoplasma non immune, rubella immune, hepatitis $B$ surface antigen negative and group B Streptococcus status unknown. Mother's obstetric history is remarkable for a previous preterm delivery at $35^{+6}$ weeks of gestation of an IUGR baby girl with a birth weight (BW) of 1500 grams.

The mother presented two days prior to delivery because of an absent diastolic flow noted 3 weeks earlier. She was admitted for potential delivery after diagnosis of severe IUGR and anhydramnios of a female fetus with growth arrest. She was given betamethasone for fetal lung maturity and magnesium sulfate for neuroprotection. Her thrombophilia workup revealed low anti thrombin III activity and low protein S activity [59\% (N: 83$128 \%$ ] and < 12\% (N: 65-140\%), respectively], indicating an increased risk for thrombosis, which explains the severe IUGR.

The infant was delivered by C-section for persistent absent diastolic flow in the umbilical artery. At birth, the infant was hypotonic with

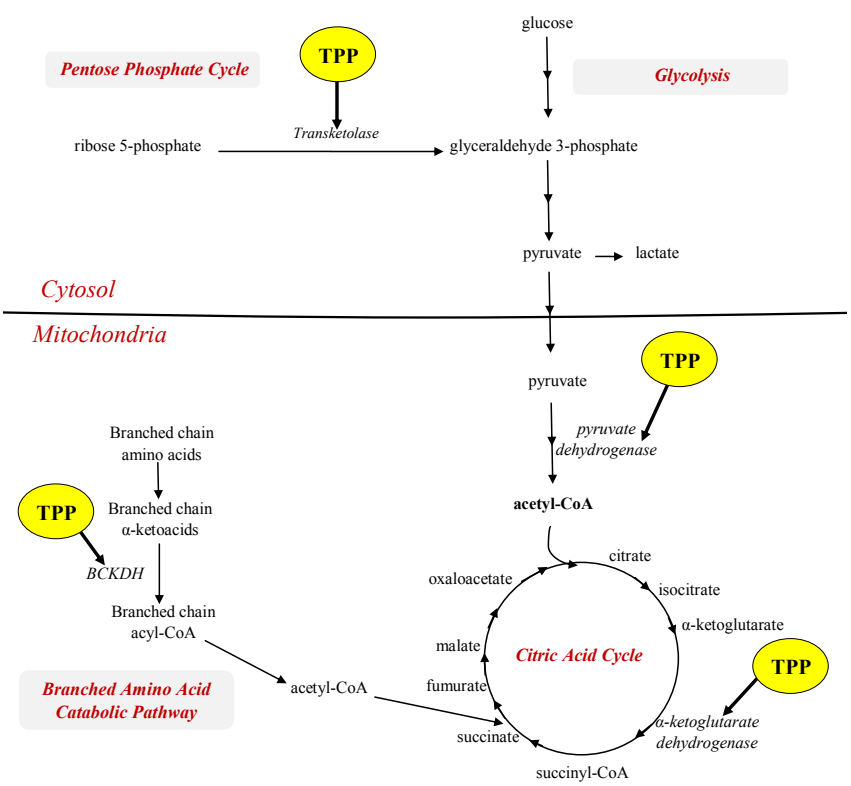

Fig.1: Thiamine pyrophosphate is the cofactor of pyruvate dehydrogenase, branched-chain alpha-keto acids dehydrogenase, transketolase, and alpha-ketoglutarate dehydrogenase. These enzymes are involved in acetyl coenzyme A formation, in branched-chain amino acids catabolism, in connecting the pentose phosphate pathway to glycolysis, and in Krebs cycle, respectively. BCKDH: branched chain ketoacid dehydrogenase complex; CoA: coenzyme A; TPP: thiamin pyrophosphate.

minimal respiratory effort; after initial steps of resuscitation, she was provided respiratory support with non-invasive positive pressure ventilation (NIPPV). Apgar scores were 8 and 8 at 1 and 5 minutes respectively. At day of life 1, the infant was hemodynamically stable, on NIPPV in room air and was started on total parenteral nutrition (TPN) by 21 hours of life. Her initial complete blood count $(C B C)$ showed a total white blood cell (WBC) count of 20,700/cu.mm with corrected WBC of 4700; while the absolute neutrophil count (ANC) was 6210/cu.mm with corrected ANC of 1410. Her platelet count at birth was $120,000 /$ 
cu.mm, and hemoglobin was $15.9 \mathrm{~g} / \mathrm{dL}$. Table 1 illustrates the glucose infusion rate (GIR), the amount of proteins and lipids provided in TPN during the first week of life and the corresponding calories. GIR was increased at day of life (DOL) 2 to correct hypoglycemia with dextro-stick of $40 \mathrm{mg} / \mathrm{dL}$. By DOL 4, GIR was increased to provide higher caloric intake; however it was decreased one day later in response to hyperglycemia (dextro-stick: $250 \mathrm{mg} /$ $\mathrm{dL}$ ). Thiamine maintenance dose of $0.25 \mathrm{mg} / \mathrm{kg}$ was part of the routine vitamins added in the TPN since the initiation of TPN at DOL 1, in addition to 0.5 $\mathrm{mg} / \mathrm{kg}$ of vitamin $B 2,4 \mathrm{mg} / \mathrm{kg}$ of nicotinamide, 0.5 $\mathrm{mg} / \mathrm{kg}$ of vitamin $\mathrm{B} 6,11 \mathrm{mg} / \mathrm{kg}$ of vitamin C, 6.0 $\mu \mathrm{g} / \mathrm{kg}$ of biotin, $40 \mu \mathrm{g} / \mathrm{kg}$ of folic acid and $0.5 \mu \mathrm{g} /$ $\mathrm{kg}$ of cyanocobalamine.

Due to the presence of respiratory distress, trophic feeding with exclusive breast milk was not introduced until DOL 3. Electrolytes imbalance with hypokalemia, decrease in phosphorus and magnesium levels [Fig.2a,2b] were noted after DOL 3. Hypokalemia was refractory to correction with intravenous (IV) supplementation. IV potassium requirement reached $8 \mathrm{meq} / \mathrm{kg} /$ day at DOL 6 , then was decreased gradually after thiamine infusion [Fig.2c].

At DOL 5, metabolic acidosis with $\mathrm{pH}$ of 7.26 and base deficit 10 was noted in addition to new onset neutropenia (ANC 1368) and thrombocytopenia (platelets 96,000). Since an umbilical venous line was in place, central line sepsis was suspected and the infant was started on vancomycin and meropenem after taking blood and urine cultures. All cultures were reported negative. In view of the electrolytes disturbances, along with metabolic acidosis, neutropenia and thrombocytopenia, refeeding syndrome with thiamine deficiency was suspected. A thiamine level could not be obtained as the measurement was not available in the country. Within 24 hours of receiving thiamine replacement at a dose of $2 \mathrm{mg} / \mathrm{kg}$ intravenously, the infant

\begin{tabular}{|ll|c|c|c|c|c|c|c|}
\hline & & DOL 1 & DOL 2 & DOL 3 & DOL 4 & DOL 5 & DOL 6 & DOL 7 \\
\hline GIR & $(\mathrm{mg} / \mathrm{kg} / \mathrm{min})$ & 6 & 10 & 10 & 11.5 & 8 & 8 & 10.3 \\
\hline Proteins & $(\mathrm{g} / \mathrm{kg} / \mathrm{day})$ & 0 & 3 & 2 & 2 & 2.5 & 2 & 2 \\
\hline Lipids & $(\mathrm{g} / \mathrm{kg} / \mathrm{day})$ & 0 & 1 & 1.5 & 2 & 1.5 & 1.5 & 1.5 \\
\hline Calories & $(\mathrm{kcal} / \mathrm{kg} / \mathrm{day})$ & 30 & 71 & 70 & 95 & 63 & 60.5 & 72 \\
\hline
\end{tabular}

Table 1: Total parenteral nutrition, glucose infusion rate $(G I R)(\mathrm{mg} / \mathrm{kg} / \mathrm{min})$ and daily total caloric intake during the first week of life.

(a)

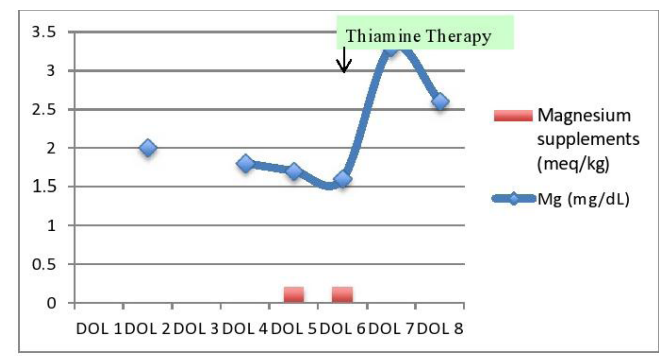

(b)

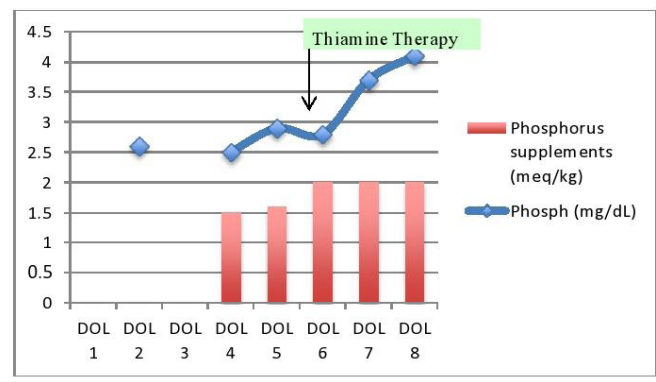

(c)

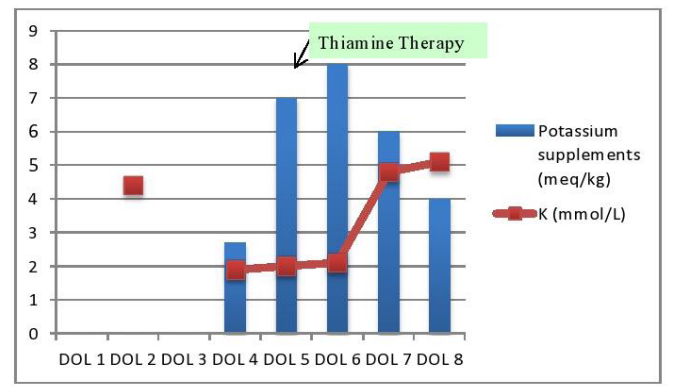

Fig.2:(a) Magnesium pattern during first week of life, and rapid decrease in the amount of supplementation following thiamine therapy, (b) Phosphorus pattern during first week of life, and the amount of phosphorus supplementation relative to thiamine therapy, (c) Potassium pattern during first week of life, and rapid decrease in the amount of supplementation following thiamine therapy. 
improved dramatically; this was accompanied by correction of the electrolytes imbalance [Fig.2], thrombocytopenia and neutropenia. The correction of metabolic acidosis was achieved 5 days after the initiation of thiamine therapy, with an increase of $\mathrm{pH}$ to 7.3 and base deficit to plus 5 .

Thiamine replacement therapy of $2 \mathrm{mg} /$ $\mathrm{kg} /$ day IV was continued for 2 more consecutive days as per the World Health Organization (WHO) guidelines for thiamine deficiency [14]. It was followed by prophylactic dose of $0.25 \mathrm{mg} / \mathrm{kg} /$ day per day via TPN. The infant remained stable thereafter with no further electrolytes disturbances. She was discharged at 3-months of age with a corrected age of $41^{+1}$ weeks of gestation and a discharge weight of $2 \mathrm{~kg}$. At her one year follow up she had appropriate development for her age and as expected, she remained below the $3^{\text {rd }}$ percentile for her corrected age.

\section{Discussion}

Placental insufficiency secondary to maternal hypercoagulable state is one of the classic causes of IUGR. In our case, the infant had severe IUGR reflecting a chronic state of malnutrition; therefore, similar to the cases of starvation, refeeding syndrome can occur upon initiation of enteral or parenteral nutrition. Refeeding syndrome has been well described in VLBW infants with IUGR [7]. Hypokalemia, hypophosphatemia, and hypomagnesemia occur upon initiation of feeding. It is the result of increased glucose metabolism leading to excess use of phosphorus for ATP production, depletion of magnesium, hypokalemia from intracellular shifts secondary to increased insulin secretion, and hyperglycemia in a system adapted to fat metabolism [7].

In addition to electrolytes imbalance, refeeding syndrome is accompanied by vitamin deficiencies, thiamine in particular [7]. Thiamine has a short half-life, is not stored in appreciable amounts and cell stores are usually depleted within 20 days of malnutrition. Abrupt increase of carbohydrate oxidation may precipitate acute deficiency because it accelerates the consumption of residual thiamine stores, [8] hence the manifestations of thiamine deficiency during the refeeding syndrome.

Thiamine deficiency presents with several manifestations including beriberi, Wernicke's encephalopathy, acute thiamine deficiency syndrome (ATD) and cardiovascular shock [12]. In neonates, ATD presenting as severe lactic acidosis was reported in a large series of neonates with bowel problems due to prematurity, who had received 10 30 days of TPN without thiamine supplementation. In most of the cases (9/11), lactic acidosis was refractory to massive doses of bicarbonate and six out of 11 died before thiamine replacement. Blood thiamine concentration was found to be significantly reduced in the 4 cases that were tested. In 5 cases, treatment with IV thiamine led to resolution of lactic acidosis and other manifestations of ATD [12]. In older children, Maiorana et al. described two patients aged 5 month-old and 9 year-old with leukemia who were given TPN for persistent vomiting and who developed acute thiamine deficiency 19 days after initiation of TPN. Both cases had electrolytes disturbances suggestive of refeeding syndrome and ATD with severe lactic acidosis and evidence of proximal renal tubular dysfunction. Review of their TPN order demonstrated lack of thiamine supplementation. Thiamine deficiency was confirmed after serum thiamine measurement in one patient and based on the characteristic laboratory findings in the other, with lactic acidosis combined with a specific urine organic acid profile. A single dose of thiamine led to rapid resolution of the tubular dysfunction and normalization of acidosis and electrolytes imbalance in both patients [8].

Thiamine plays a part in many different aspects of energy metabolism [Fig.1]. When 
thiamine is deficient, a combined enzymatic defect occurs causing Krebs cycle and ATP synthesis impairment. As a result, thiamine deficiency may cause disruption of renal tubular function, resulting in decreased reabsorption of electrolytes as a consequence of impaired tubular ATP-dependent transport systems [8]. Through transketolase activity, thiamine plays a pivotal role in protecting against oxidative stress, thus in thiamine deficiency the insufficient ATP generation combined with toxic effects of reactive oxygen species can lead to acute tubular necrosis through an ischemia-reperfusion injury [8]. In our reported case, the metabolic acidosis and the electrolytes imbalance could be attributed to tubular impairment in its early phase caused by thiamine deficiency, instead of a shifting from extracellular to intracellular compartment. Unfortunately, the thiamine level was not measured, as it is not available in our institution.

In extremely premature infants, it is generally recommended to initiate TPN with 3-4 $\mathrm{g} / \mathrm{kg} /$ day of amino acids as soon as possible to avoid the consequences of the catabolic state due to premature birth [15]; however in severe IUGR infants, chronic in-utero malnutrition should be kept in mind and the possibility of refeeding syndrome should be considered in case of unexplained electrolytes disturbances with metabolic acidosis. Moreover, supplementation with higher amount of thiamine in TPN should be discussed.

\section{Conclusion}

During initiation of feeding, severe IUGR infants should be carefully monitored for electrolytes abnormalities. Thiamine deficiency should be highly suspected whenever electrolytes imbalance is present in combination with lactic acidosis, neutropenia and thrombocytopenia. This potentially life threatening condition responds dramatically to thiamine administration when promptly recognized.

\section{References}

1. Parli SE, Ruf KM, Magnuson B. Pathophysiology, treatment, and prevention of fluid and electrolyte abnormalities during refeeding syndrome. Journal of Infusion Nursing. 2014;37(3):197202.

2. Byrnes MC, Stangenes J. Refeeding in the ICU: an adult and pediatric problem. Current Opinion in Clinical Nutrition and Metabolic Care. 2011 ; 14(2):186-192.

3. Fuentebella J, Kerner JA. Refeeding syndrome. Pediatric clinics of North America. 2009;56(5): $1201-1210$.

4. Gentile MG. Enteral nutrition for feeding severely underfed patients with anorexia nervosa. Nutrients. $2012 ; 4(9): 1293-1303$.

5. Mizumoto $H$, Mikami M, Oda $H$, Hata D. Refeeding syndrome in a small-for-dates micropreemie receiving early parenteral nutrition. Pediatrics International. 2012;54(5):715-717.

6. Moltu SJ, Strommen K, Blakstad EW, Almaas AN, Westerberg AC, Brakke K, et al. Enhanced feeding in very-low-birth-weight infants may cause electrolyte disturbances and septicemia-a randomized, controlled trial. Clinical Nutrition. 2013;32(2):207-212.

7. Ross JR, Finch C, Ebeling M, Taylor SN. Refeeding syndrome in very-low-birth-weight intrauterine growth-restricted neonates. Journal of Perinatology. 2013;33(9):717-720.

8. Maiorana A, Vergine G, Coletti V, Luciani $M$, Rizzo C. Acute thiamine deficiency and refeeding syndrome: Similar findings but different pathogenesis. Nutrition. 2014;30/78):948-952.

9. Hakan N, Aydin M, Dilli D, Zenciroglu A, Okumus $N$. Transient hyperinsulinemia may be responsible from electrolyte abnormalities of refeeding syndrome seen in very low birth weight infants with intrauterine growth-restriction. Journal of Perinatology. 2014;34(3):247.

10. Stanga Z, Brunner A, Levenberger M, Grimble 
RF, Shenkin A, Allison SP, et al. Nutrition in clinical practice-the refeeding syndrome: illustrative cases and guidelines for prevention and treatment. European Journal of Clinical Nutrition. 2008;62(6):687-694.

11. Walmsley RS. Refeeding syndrome: screening, incidence, and treatment during parenteral nutrition. Journal of Gastroenterology and Hepatology. 2013;28 Suppl 4:113-117.

12. Thauvin-Robinet $C$, Faivre L, Barbier $M L$, Chevret L, Bourgeois J, Netter JC, et al. Severe lactic acidosis and acute thiamin deficiency: a report of 11 neonates with unsupplemented total parenteral nutrition. Journal of Inherited Metabolic Disease. 2004;27(5):700-704.

13. Oguz SS, Ergenekon E, Tumer L, Koc E, Turan
$O$, Onal $E$, et al. A rare case of severe lactic acidosis in a preterm infant: lack of thiamine during total parenteral nutrition. Journal of Pediatric Endocrinology \& Metabolism. 2011 ;24(9-10):843-845.

14. World Health Organization UNHCR. Thiamine deficiency and its prevention and control in major emergencies. World Health Organization 1999. Available from: http://www.who.int/nutrition/ publications/en/thiamine_in_emergencies_eng. pdf. Accessed on 19 February 2016.

15. de Boo HA, Harding JE. Protein metabolism in preterm infants with particular reference to intrauterine growth restriction. Archives of Disease in Childhood Fetal and Neonatal Edition. 2007;92(4):F315-319. 